This is the final accepted manuscript of:

A. Melli, M. Melosso, N. Tasinato, et al. ROTATIONAL AND INFRARED SPECTROSCOPY OF ETHANIMINE: A ROUTE TOWARDS ITS ASTROPHYSICAL AND PLANETARY DETECTION. Astrophys. J. 855, 123 (2018); DOI: 10.3847/1538-4357/aaa899

Available at: https://doi.org/10.3847/1538-4357/aaa899

(C) 2018. The American Astronomical Society. 
Draft Version January 5, 2018

Typeset using LATEX twocolumn style in AASTeX61

\title{
ROTATIONAL AND INFRARED SPECTROSCOPY OF ETHANIMINE: A ROUTE TOWARDS ITS ASTROPHYSICAL AND PLANETARY DETECTION
}

Alessio Melli, ${ }^{1}$ Mattia Melosso, ${ }^{1}$ Nicola Tasinato, ${ }^{2}$ Giulio Bosi, ${ }^{1}$ Lorenzo Spada,,${ }^{1,2}$ Julien Bloino,,${ }^{2,3}$ Marco Mendolicchio, ${ }^{2}$ Luca Dore, ${ }^{1}$ Vincenzo Barone, ${ }^{2}$ and Cristina Puzzarini ${ }^{1}$

${ }^{1}$ Dipartimento di Chimica "Giacomo Ciamician", Università di Bologna, Via Selmi 2, 40126, Bologna (Italy).

${ }^{2}$ Scuola Normale Superiore, Piazza dei Cavalieri 7, 56126, Pisa (Italy).

${ }^{3}$ Consiglio Nazionale delle Ricerche, Istituto di Chimica dei Composti OrganoMetallici (ICCOM-CNR), UOS di Pisa, Area della Ricerca CNR, Via G. Moruzzi 1, 56124, Pisa (Italy).

\begin{abstract}
Ethanimine, a possible precursor of amino acids, is considered an important prebiotic molecule and thus may play important roles in the formation of biological building-blocks in the interstellar medium. In addition, its identification in Titan's atmosphere would be important for understanding the abiotic synthesis of organic species. An accurate computational characterization of the molecular structure, energetics and spectroscopic properties of the $E$ and $Z$ isomers of ethanimine, $\mathrm{CH}_{3} \mathrm{CHNH}$, has been carried out by means of a composite scheme based on coupled-cluster techniques, which also accounts for extrapolation to the complete basis-set limit and core-valence correlation correction, combined with density functional theory for the treatment of vibrational anharmonic effects. By combining the computational results with new millimeter-wave measurements up to $300 \mathrm{GHz}$, the rotational spectrum of both isomers can be accurately predicted up to $500 \mathrm{GHz}$. Furthermore, our computations allowed us to revise the infrared spectrum of both $E$ - and $Z-\mathrm{CH}_{3} \mathrm{CHNH}$, thus predicting all fundamental bands with high accuracy.
\end{abstract}

Keywords: ISM: molecules - line: identification - methods: data analysis - molecular data - planets and satellites: atmospheres

Corresponding author: Cristina Puzzarini

cristina.puzzarini@unibo.it

Corresponding author: Nicola Tasinato

nicola.tasinato@sns.it 


\section{INTRODUCTION}

The starting point for the development of astrochemical models is the knowledge whether a molecule is present in the astronomical environment considered, with the (radio)astronomical observation of spectroscopic signatures providing the unequivocal proof of its presence (Tennyson 2005). So far, almost 200 molecules have been detected in the interstellar medium (ISM) and circumstellar shells (CDMS: Müller et al. 2001, 2005), and most of them have been identified by means of their rotational transitions (Herbst \& van Dishoeck 2009; Tielens 2013). Indeed, the rotational signatures can be considered the fingerprints of the molecule under consideration, thus allowing its unequivocal identification, even of one isotopic species from another (Gordy \& Cook 1984). However, infrared (IR) spectroscopy can play an important role because it is suitable for retrieving the chemical composition of planetary atmospheres (e.g. Tinetti et al. 2013). The required rotational and vibrational transition frequencies are accurately obtained from laboratory measurements that are increasingly assisted by quantum-chemical calculations to guide and support the spectral recording and analysis. On general grounds, computational chemistry plays a fundamental role in obtaining accurate energetic, structural and spectroscopic parameters (Puzzarini et al. 2010b, 2014; Barone et al. 2015a; Puzzarini 2017). In this work, the rotational and infrared spectra of both $E$ and $Z$ isomers of ethanimine, $\mathrm{CH}_{3} \mathrm{CHNH}$, have been accurately characterized by means of state-of-the-art computational methodologies, with new millimeter-wave measurements also carried out to further improve the predictive capabilities of our rotational-spectroscopy characterization.

The importance of ethanimine is related to its prebiotic potential as possible precursor of amino acids (Quan et al. 2016), like alanine, by reaction with HCN and $\mathrm{H}_{2} \mathrm{O}$, or with formic acid (Woon 2002; Elsila et al. 2007; Loomis et al. 2013). From an astrophysical point of view, $\mathrm{CH}_{3} \mathrm{CHNH}$ is classified as a complex organic molecule (COM); the detection of COMs and in particular those related to the formation of biomolecules is one of the most important themes in astrochemistry (e.g. Herbst \& van Dishoeck 2009; Barone et al. 2015b; Puzzarini 2017). Both isomers of ethanimine have been identified in the survey of Sagittarius B2 North, SgrB2(N), taken in the frame of the Green Bank Telescope (GBT) PRebiotic Interstellar MOlecule Survey (GBT PRIMOS) legacy project (Loomis et al. 2013). Furthermore, a computational work by Balucani et al. (2010) has foreseen ethanimine as one of the main products of the reaction between ethane, $\mathrm{C}_{2} \mathrm{H}_{6}$, and excited nitrogen atoms, $\mathrm{N}\left({ }^{2} \mathrm{D}\right)$, in Titan's atmosphere, which is mainly composed of nitrogen and in a small percentage of methane and other light hydrocarbons, in abundances varying with the altitude (Brown et al. 2010). In addition, Titan is the only solar system body with a molecular nitrogen-based atmosphere, like the primitive Earth (Balucani et al. 2010), which explains its importance in the study of prebiotic chemistry. Of interest to the present study, the Composite Infrared Spectrometer (CIRS) and the Ion and Neutral Mass Spectroscopy (INMS) aboard Cassini have already confirmed the presence of ethane and methanimine in Titan's atmosphere (Shemansky et al. 2005; Brown et al. 2010). In the context of this study, it is important to note that the latter molecule plus the $\mathrm{CH}_{3}$ radical and ethanimine plus $\mathrm{H}$ are the two main reaction channels of the $\mathrm{N}\left({ }^{2} \mathrm{D}\right)+$ $\mathrm{C}_{2} \mathrm{H}_{6}$ reactive system (Balucani et al. 2010).

As far as the previous experimental works on $\mathrm{CH}_{3} \mathrm{CHNH}$ are concerned, the rotational spectra of both isomers have already been characterized up to 140 GHz (Loomis et al. 2013; Brown et al. 1980; Lovas et al. 1980). However, extrapolations from low-frequency laboratory measurements usually provide inaccurate predictions for higher frequencies and, in view of the nowadays extended astronomical observatory facilities (e.g. the Atacama Large Millimeter/submillimeter Array of radiotelescopes (ALMA) working frequency range is 31-950 GHz), the extension of the investigation of the rotational spectra for the $E$ and $Z$ isomers of ethanimine is warranted. For this reason, our computational work has been combined with new measurements in the millimeter-wave regime up to $300 \mathrm{GHz}$. Concerning vibrational spectroscopy, low-resolution gas-phase (Hashiguchi et al. 1984) and matrix (Stolkin et al. 1977) infrared spectra have been experimentally investigated, with both studies providing either incomplete or contradictory results. From a computational point of view, fundamental frequencies were only reported at a low level of theory and within the harmonic approximation (Durig et al. 2010). Prompted by the limitations of both experimental and computational previous studies, in this work we have revised the infrared spectrum at a higher level of theory, with a complete account of anharmonicity.

In summary, to extend the spectroscopic characterization of both isomeric forms of $\mathrm{CH}_{3} \mathrm{CHNH}$, an accurate computational study of the energetics as well as structural and spectroscopic parameters has been carried out, complemented by new experimental measurements of the rotational spectrum. Computationally, a composite scheme has been employed, which combines coupledcluster techniques with extrapolation to the complete basis-set limit and consideration of core-correlation con- 
tributions. Vibrational effects, including anharmonicity, have been accounted for by means of density functional theory. For both isomers, the simulated rotational, up to $500 \mathrm{GHz}$, and infrared, including all fundamentals, spectra are very accurate, thus increasing the opportunity to directly guide astronomical searches.

\section{COMPUTATIONAL METHODOLOGY}

The quantum-chemical calculations described in the following have been carried out with the Gaussian16 suite of programs for quantum chemistry (Frisch et al. 2016) and the CFOUR program package (Stanton et al. 2008), with the former employed for the density functional theory (DFT) computations and the latter for the coupled-cluster (CC) ones.

The first step for obtaining a reliable simulation of the rotational spectra of both ethanimine isomers is the accurate determination of their equilibrium structures. For this purpose, the well-tested "CCSD $(\mathrm{T}) / \mathrm{CBS}+\mathrm{CV}$ " energy-gradient composite scheme (Heckert et al. 2005, 2006), for which the expected accuracy for bond lengths and angles is 0.001-0.002 $\AA$ and 0.05-0.1 degrees, respectively (see Heckert et al. 2005, 2006; Puzzarini et al. 2008, 2010b; Barone et al. 2013), has been employed. This scheme is based on coupled-cluster techniques, in detail, on the $\mathrm{CC}$ singles and doubles approximation (CCSD) augmented by a perturbative treatment of triples $(\mathrm{CCSD}(\mathrm{T}))$ (Raghavachari et al. 1989). It accounts for the following contributions: $(i)$ the HartreeFock self-consistent field (HF-SCF) energy gradient extrapolated to the complete basis-set (CBS) limit by means of the 3-point formula by Feller (1993), with the cc-pVTZ, cc-pVQZ and cc-pV5Z basis sets (Dunning 1989) considered; (ii) the valence CCSD(T) correlation energy gradient extrapolated to CBS limit using the 2-point $\mathrm{n}^{-3}$ extrapolation formula (Helgaker et al. 1997) employing the cc-pVnZ basis sets, with $\mathrm{n}=\mathrm{T}$, Q; (iii) the core-valence $(\mathrm{CV})$ correlation energy gradient correction obtained as difference of all electrons (all-) and frozen core (fc-) $\operatorname{CCSD}(\mathrm{T})$ calculations using the cc-pCVTZ basis set (Woon \& Dunning 1995; Dunning 1989).

The vibrational ground-state rotational constants, needed for the prediction of the rotational spectrum, have been obtained by combining the equilibrium rotational constants, straightforwardly derived from the $\operatorname{CCSD}(\mathrm{T}) / \mathrm{CBS}+\mathrm{CV}$ geometries, with the corresponding vibrational corrections, computed using the double hybrid B2PLYP functional (Grimme 2006) augmented for dispersion corrections according to the DFT-D3 scheme (Grimme et al. 2011) with Becke-Johnson damping (Goerigk \& Grimme 2011), namely B2PLYP-D3BJ, in conjunction with the maug-cc-pVTZ basis set (Papajak et al. 2009) in which $d$ functions on hydrogen atoms were removed (this basis set will be referred to in the following as maug-cc-pVTZ- $d \mathrm{H}$ ). Vibrational corrections to rotational constants require the evaluation of a cubic force field, which - as a byproduct - gives access to the computation of the sextic centrifugal distortion constants. The quartic ones require instead the determination of a harmonic force field, which has been evaluated at a higher level of theory, i.e. employing the $\operatorname{CCSD}(\mathrm{T})$ method in conjunction with the cc-pCVQZ basis set (Woon \& Dunning 1995; Dunning 1989), with all electrons correlated. To complete the characterization of the rotational spectrum, nitrogen quadrupole coupling constants and the spectroscopic parameters related to the methyl internal rotation are needed. To evaluate the former constants, the electric field gradient (EFG) tensor has been evaluated for both isomers at the $\operatorname{CCSD}(\mathrm{T}) / \mathrm{cc}$-pCVQZ level using the $\operatorname{CCSD}(\mathrm{T}) / \mathrm{CBS}+\mathrm{CV}$ geometry and augmented by vibrational corrections at the B2PLYP-D3BJ/maug-ccpVTZ- $d$ H level. For all details related to the conversion of the EFG elements to the nuclear quadrupole coupling constants, the reader is for example referred to Puzzarini et al. (2010b); Puzzarini (2013).

The characterization of the methyl internal rotation requires first of all an accurate evaluation of the energetics of the system. For this purpose, the potential energy surface (PES) has been investigated at the B2PLYP-D3BJ/maug-cc-pVTZ- $d \mathrm{H}$ level, thus locating the two minima, the $E-Z$ isomerization transition state $\left(\mathrm{TS}_{E-Z}\right)$, and for each isomer, the transition state for the methyl internal rotation $\left(\mathrm{TS}_{E}\right.$ and $\mathrm{TS}_{Z}$ for the $E$ and $Z$ isomers, respectively). The internal rotation of the $-\mathrm{CH}_{3}$ group relative to the $-\mathrm{CHNH}$ frame leads to three equivalent configurations; the potential energy along the torsional angle $(\angle \mathrm{H} 8-\mathrm{C} 1-\mathrm{C} 2-\mathrm{N} 3=$ $\varphi)$ has thus periodicity 3 and can be simply written as $V_{3} \cos (3 \varphi)$, where $V_{3}$ is the energy barrier. The B2PLYP-D3BJ/maug-cc-pVTZ- $d \mathrm{H}$ level of theory has been chosen because it has proven to provide accurate reference structures for energetic calculations (see, for example, Barone et al. 2015a; Vazart et al. 2016; Spada et al. 2017). On top of B2PLYP-D3BJ/maug-cc-pVTZ$d \mathrm{H}$ equilibrium and transition-state geometries, the energetics has been evaluated at the CCSD(T)/CBS+CV level, with the relative energy values then augmented by zero-point energy (ZPE). The latter has been obtained at the anharmonic level using the non-resonant formulation of second-order vibrational perturbation theory, VPT2 (Mills 1972; Schuurman et al. 2005; Piccardo et al. 2015), based on B2PLYP-D3BJ/maug-cc-pVTZ- 
$d \mathrm{H}$ data. Hence, the barrier governing the $E-Z$ isomerization and the $V_{3}$ barrier have been calculated as energy differences between the transition states and the corresponding minima. To complete the description of the parameters involving the internal rotation, the moment of inertia of the methyl rotor, $I_{\alpha}$, has been evaluated. In analogy to rotational constants, the equilibrium moment of inertia, straightforwardly derived from the $\mathrm{CCSD}(\mathrm{T}) / \mathrm{CBS}+\mathrm{CV}$ geometry, has been corrected for the vibrational contribution at the B2PLYP-
D3BJ/maug-cc-pVTZ- $d \mathrm{H}$ level. More precisely, in order to obtain the effective $I_{\alpha}$ corresponding to the vibrational ground state, a least-squares fit of the moments of inertia of all mono-substituted isotopic species of $\mathrm{CH}_{3} \mathrm{CHNH}$, evaluated by combining the equilibrium and vibrational contributions, has been carried out with the MSR software (Mendolicchio et al. 2017). The effective $I_{\alpha}$ corresponds to the so-called vibrationally averaged structure $r_{0}$ (Gordy \& Cook 1984).

Table 1. Observed Transition Frequencies of $E$ - and $Z$-ethanimine.

\begin{tabular}{ccccccccccc}
\hline \hline Isomer & $\mathrm{A} / \mathrm{E}$ & $J^{\prime}$ & $K_{a}^{\prime}$ & $K_{c}^{\prime}$ & $J^{\prime \prime}$ & $K_{a}^{\prime \prime}$ & $K_{c}^{\prime \prime}$ & $\begin{array}{c}\text { Obs. frequency } \\
(\mathrm{MHz})\end{array}$ & $\begin{array}{c}\text { Uncertainty } \\
(\mathrm{MHz})\end{array}$ & $\begin{array}{c}\text { Obs-calc } \\
(\mathrm{MHz})\end{array}$ \\
\hline$(E)$ & $\mathrm{A}$ & 15 & 0 & 15 & 14 & 1 & 14 & 257731.650 & 0.040 & -0.039 \\
$(E)$ & $\mathrm{E}$ & 15 & 0 & 15 & 14 & 1 & 14 & 257755.704 & 0.040 & 0.010 \\
$(E)$ & $\mathrm{E}$ & 15 & 0 & 15 & 14 & 0 & 14 & 268872.179 & 0.040 & -0.057 \\
$(E)$ & $\mathrm{A}$ & 15 & 0 & 15 & 14 & 0 & 14 & 268879.762 & 0.040 & -0.025 \\
$(E)$ & $\mathrm{A}$ & 16 & 0 & 16 & 15 & 1 & 15 & 276962.242 & 0.040 & -0.052 \\
$(E)$ & $\mathrm{E}$ & 16 & 0 & 16 & 15 & 1 & 15 & 276981.826 & 0.040 & 0.042 \\
$(Z)$ & $\mathrm{E}$ & 15 & 1 & 15 & 14 & 1 & 14 & 265661.991 & 0.040 & 0.006 \\
$(Z)$ & $\mathrm{A}$ & 15 & 1 & 15 & 14 & 1 & 14 & 265666.519 & 0.040 & -0.036 \\
$(Z)$ & $\mathrm{E}$ & 15 & 0 & 15 & 14 & 0 & 14 & 267268.829 & 0.040 & 0.056 \\
$(Z)$ & $\mathrm{A}$ & 15 & 0 & 15 & 14 & 0 & 14 & 267278.988 & 0.040 & -0.073 \\
$(Z)$ & $\mathrm{E}$ & 17 & 0 & 17 & 16 & 0 & 16 & 301598.448 & 0.040 & 0.069 \\
$(Z)$ & $\mathrm{A}$ & 17 & 0 & 17 & 16 & 0 & 16 & 301607.845 & 0.040 & -0.022 \\
\hline
\end{tabular}

Note-This table is available in its entirety in machine-readable form.

Ethanimine is a non-planar molecule of $C_{s}$ symmetry, with the $c$ axis being perpendicular to the symmetry $a b$ plane. Its fundamental vibrations therefore belong to the $\mathbf{A}^{\prime}\left(\nu_{1}-\nu_{12}\right)$ or $\mathbf{A}^{\prime \prime}$ $\left(\nu_{13}-\nu_{18}\right)$ irreducible representations. The investigation of the IR spectrum is based on harmonic and anharmonic force-field determinations. To provide accurate predictions, we relied on a hybrid CC/DFT approach, which is well documented in the literature and is expected to predict fundamental transitions with an accuracy of about $5-10 \mathrm{~cm}^{-1}$ (see, for example, Puzzarini et al. 2010a, 2011; Barone et al. 2013, 2014, 2015a). This approach combines the all-CCSD(T)/ccpCVQZ harmonic component with anharmonic contributions evaluated at the DFT level. As implemented in CFOUR, the harmonic part has been obtained using analytic-second derivatives of the energy (Gauss \& Stan- ton 1997). The cubic and semi-diagonal quartic force constants have been evaluated by numerical differentiation of analytical second-order derivatives (Schneider \& Thiel 1989) at the B2PLYP-D3BJ/maug-cc-pVTZ- $d \mathrm{H}$ level of theory. The vibrational spectra have then been simulated beyond the double-harmonic approximation, making use of VPT2 applied to the hybrid force field. For IR intensities beyond the double harmonic approximation, second- and semi-diagonal third-order derivatives of the electric dipole moment surface have been calculated by numerical differentiation of analytic first derivatives of the electric dipole moment. These have been employed within a general formulation to compute anharmonic vibrational averages and transition properties at the second-order of perturbation theory (Barone 2005; Bloino \& Barone 2012). To tackle the problem of resonances present within the VPT2 approach, the 
GVPT2 (Generalized VPT2) protocol has been adopted (Barone 2005; Bloino et al. 2016). According to it, the (near-)singular terms are first removed from the perturbative expressions (DVPT2) and the interaction between the involved states reintroduced in a second step by means of a variational approach applied to a small effective Hamiltonian (Bloino et al. 2012; Piccardo et al. 2015). All VPT2 computations have been carried out with the Gaussian suite of quantum chemical programs (Frisch et al. 2016).

\section{EXPERIMENTAL DETAILS}

Ethanimine is an unstable molecule that belongs to the family of imines. In the present work, it was produced through pyrolysis of a commercial sample of isopropylamine, $\left(\mathrm{CH}_{3}\right)_{2} \mathrm{CHNH}_{2}$, as previously suggested by Lovas et al. (1980). The vapours of the precursor were flowed inside a quartz tube heated by a 30 $\mathrm{cm}$ long tube furnace, within the same apparatus employed in the production of other unstable molecules (see, for example, Dore et al. (2012); Degli Esposti et al. (2014); Melosso et al. (2017)). The highest yield of production for both isomers was attained by setting the oven temperature at about $1150{ }^{\circ} \mathrm{C}$ and by flowing the vapours of isopropylamine at a pressure of 100 mTorr through the quartz tube, which corresponds to a pressure of 15 mTorr in the free-space glass absorption cell. The rotational spectra were recorded around $92 \mathrm{GHz}$ and in the 250-302 GHz spectral window using a millimeter/submillimeter-wave frequency-modulation spectrometer (Degli Esposti et al. 2017). The radiation sources were either a Gunn diode operating in the 85$115 \mathrm{GHz}$ range or a passive multiplier (frequency tripler, VDI) driven by the Gunn diode. The stability of the output frequency was ensured by a phase-lock loop (PLL) system with a reference signal frequency modulated at $6 \mathrm{kHz}$ and referred to a rubidium atomic clock. Phasesensitive detection at twice the frequency-modulation is employed, so that the second derivative of the spectrum is displayed. A Schottky barrier Millitech detector was used for recording the spectra at $92 \mathrm{GHz}$, while a VDI detector was employed at higher frequencies. The experimental uncertainties of our measurements mainly depend on the signal-to-noise ratio $(\mathrm{S} / \mathrm{N})$ of the spectra and were estimated to be $40-60 \mathrm{kHz}$. For both isomers, a selection of newly recorded transitions is listed in Table 1 , while the complete list together with all previous frequency values included in the fits and the corresponding residuals (i.e., the observed - calculated differences) are included in the online machine readable version of this table. The fits are discussed in Section 4. It has to be noted that, the $V_{3}$ barrier being finite, a tunneling effect occurs, which leads to a splitting of the threefold degeneracy into two levels, a nondegenerate A level and a doubly-degenerate $\mathrm{E}$ level. As a consequence, in the rotational spectrum, rotational transitions in the $\mathrm{A}$ and E torsional sublevels are observed. Figure 1 provides an example of the $\mathbf{A} / \mathbf{E}$ splittings observed in the rotational spectrum. The effect of the barrier height on them can be expressed by means of a perturbative approach. A complete description of the treatment being too long to be summarized here, the reader is referred to (Gordy \& Cook 1984).

\section{RESULTS AND DISCUSSION}

The equilibrium CCSD(T)/CBS+CV $\left(r_{e}\right)$ and vibrationally averaged $\left(r_{0}\right)$ geometrical parameters for the $Z$ and $E$ isomers are reported in Table 2. It is first of all noted that small differences in bond lengths and angles are generally observed between the two isomers, with the only noticeable exception for the $\angle \mathrm{N} 3-\mathrm{C} 2-\mathrm{C} 1$ angle, which increases by about six degrees when moving from $E$ to $Z$. This discrepancy is ascribable to the plausible formation of a weak intramolecular C-H. .N hydrogen bond in the $E$ isomer, where one $\mathrm{C}-\mathrm{H}$ bond of the methyl group is directed toward the lone pair of the nitrogen atom. For the same angle, a nearly three times smaller difference has been observed in the case of the $Z$ to $E$ isomerism of cyanomethanimine (Puzzarini 2015), for which, however, the former isomer is the most stable one by about $161.2 \mathrm{~cm}^{-1}$ (at the $\operatorname{CCSD}(\mathrm{T}) / \mathrm{CBS}+\mathrm{CV}$ level corrected for anharmonic ZPE at the CCSD(T)/ccpVTZ level, see Puzzarini (2015)). In contrast, the $Z$ isomer of ethanimine is $\sim 231.5 \mathrm{~cm}^{-1}$ higher in energy than the $E$ form at the CCSD(T)/CBS+CV level corrected for the anharmonic ZPE at the B2PLYPD3BJ/maug-cc-pVTZ- $d \mathrm{H}$ level. The two isomers of ethanimine are separated by an interconversion barrier that is as high as $9677 \mathrm{~cm}^{-1}$ with respect to the $E$ isomer (at the $\operatorname{CCSD}(\mathrm{T}) / \mathrm{CBS}+\mathrm{CV}$ level, with the ZPE obtained at the B2PLYP-D3BJ/maug-cc-pVTZ- $d \mathrm{H}$ level). The corresponding transition state shows an almost linear arrangement of the H4-N3-C2 angle (179.3 degrees at the B2PLYP-D3BJ/maug-cc-pVTZ- $d \mathrm{H}$ level), which is similar to what was found for cyanomethanimine (179.1 degrees at the fc-CCSD(T)/cc-pVTZ level). 


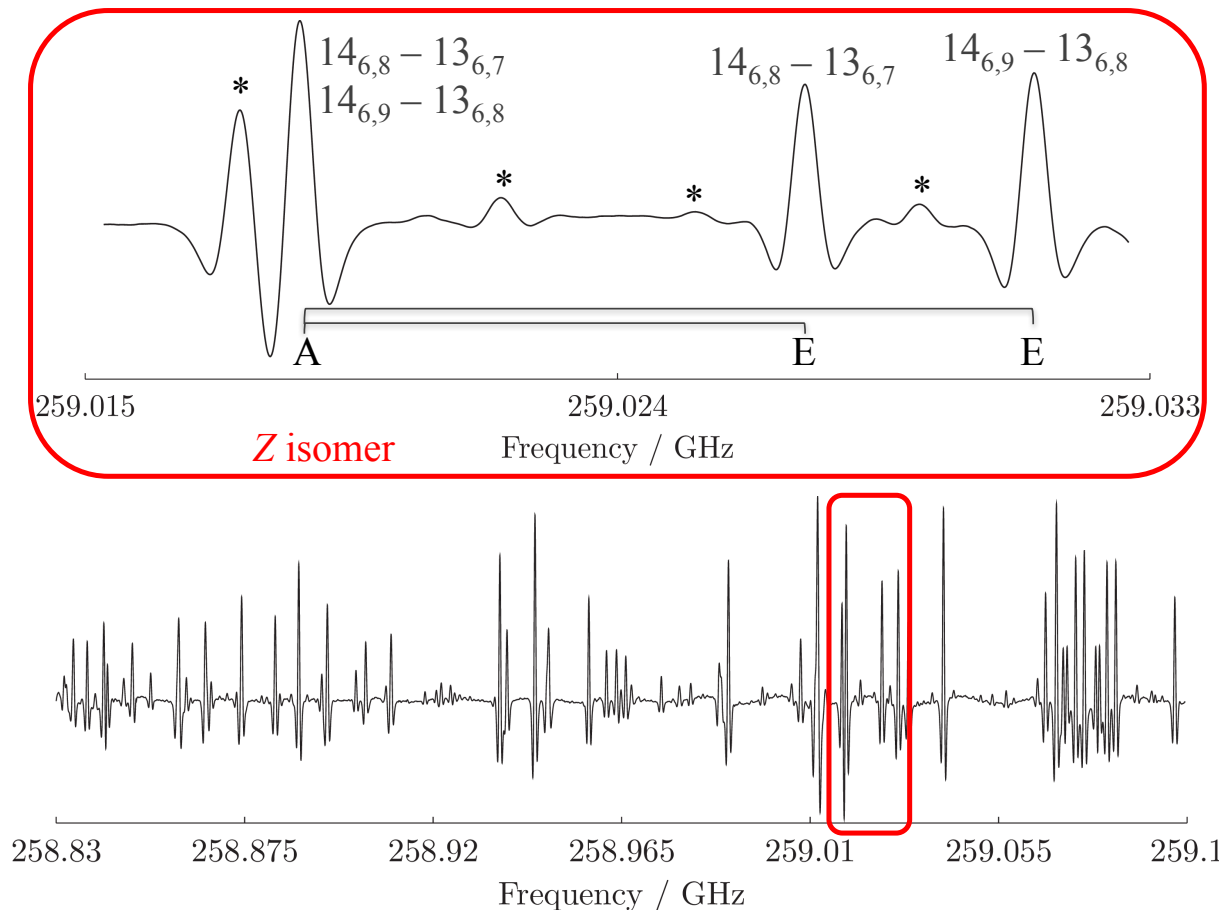

Figure 1. Small portion $(\sim 270 \mathrm{MHz})$ of the rotational spectrum recorded at $\sim 259 \mathrm{GHz}$. In the inset, the assignment of a couple of $\mathrm{A} / \mathrm{E}$ splittings for the $Z$ isomer is shown in detail; the asterisks denote non assigned transitions due to pyrolysis byproducts or vibrational excited states.

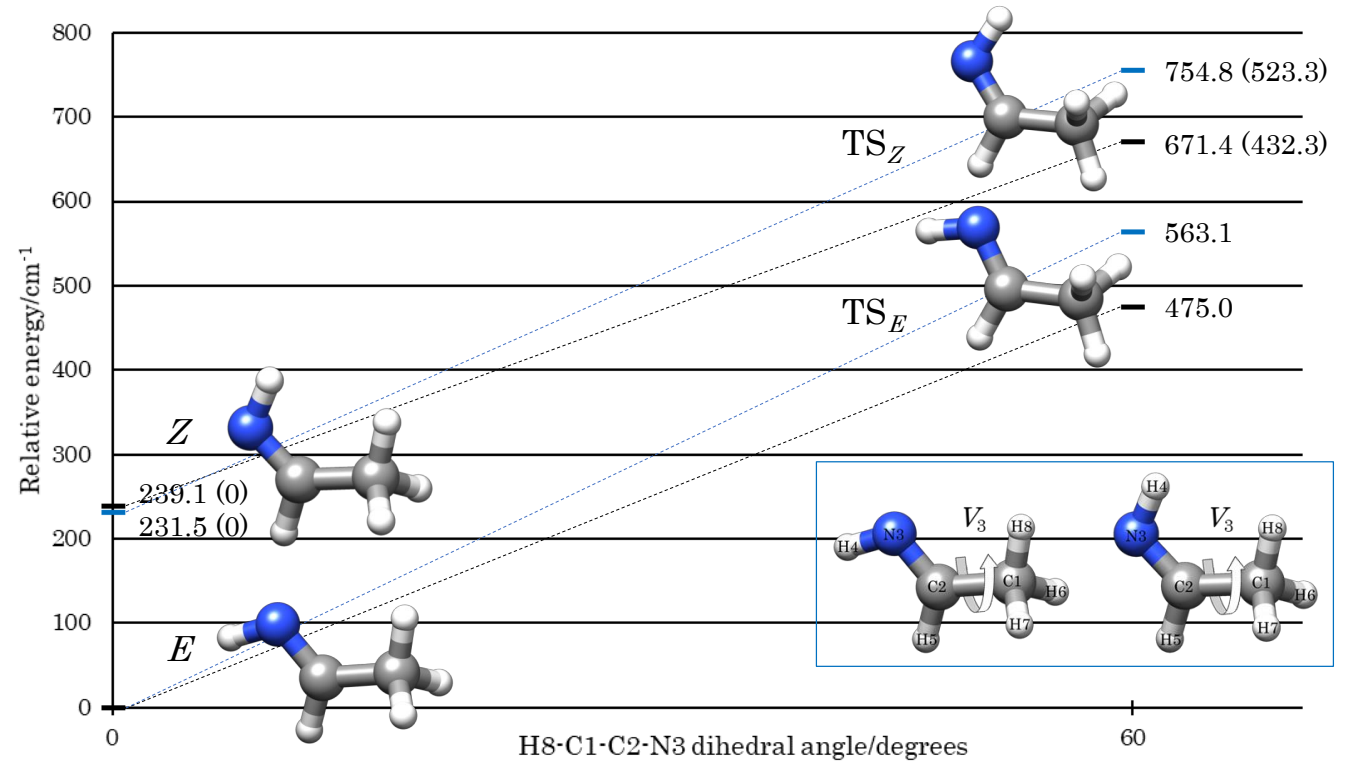

Figure 2. Equilibrium (CCSD $(\mathrm{T}) / \mathrm{CBS}+\mathrm{CV}$, in black) and ZPE-corrected (B2PLYP-D3BJ/maug-cc-pVTZ- $d \mathrm{H}$, in blue) energies for the $Z$ isomer minimum and methyl internal rotation transition states $\left(\mathrm{TS}_{E}, \mathrm{TS}_{Z}\right)$ with respect to $E-\mathrm{CH}_{3} \mathrm{CHNH}$. Values in parentheses are referred to the $Z$ isomer. 
Table 2. Equilibrium $\mathrm{CCSD}(\mathrm{T}) / \mathrm{CBS}+\mathrm{CV}\left(r_{e}\right)$ and vibrationally averaged $\left(r_{0}\right)$ structures.

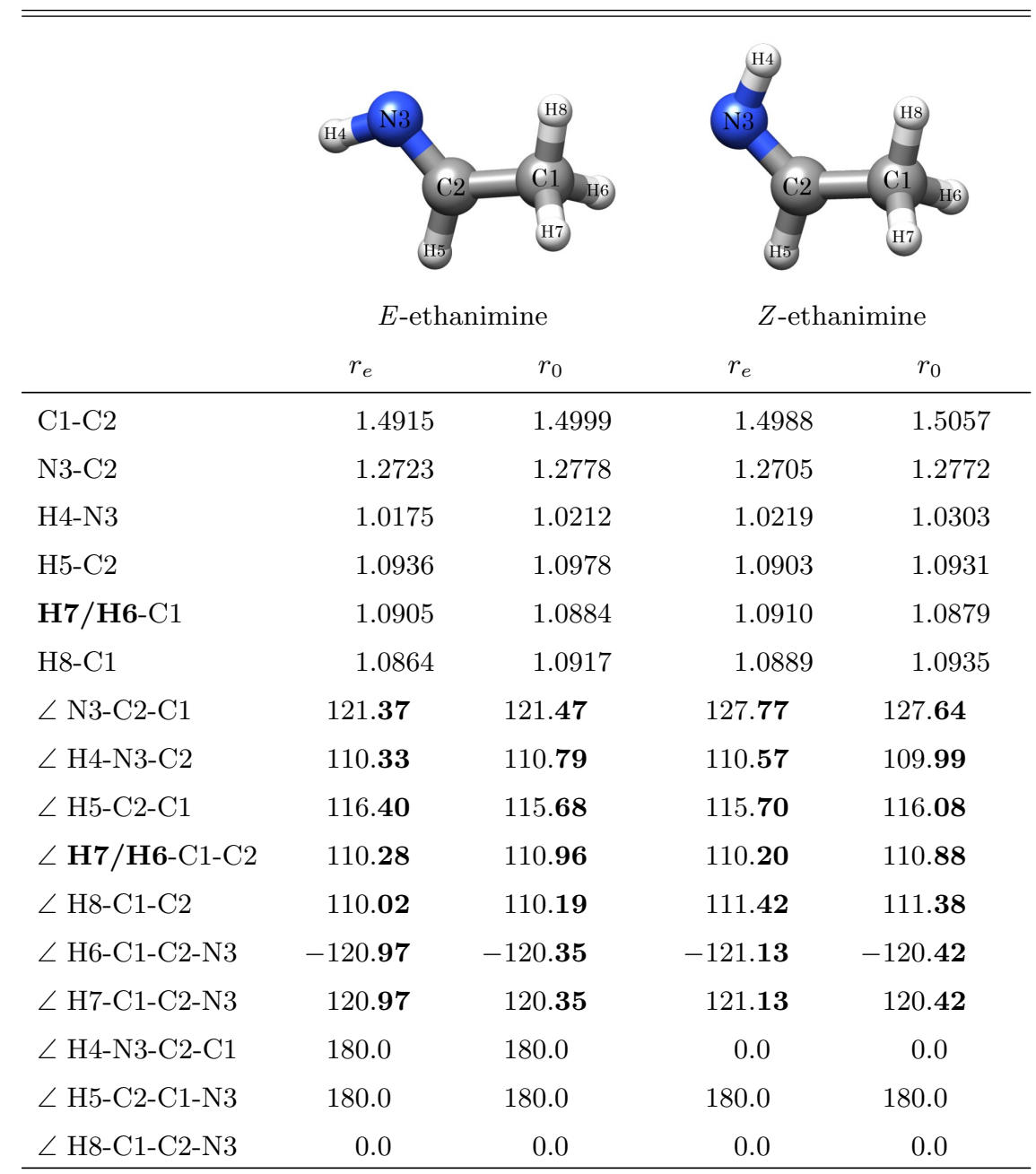

Note-Bond lengths in $\AA$, angles in degrees.

To simulate the rotational spectra of both isomers, the effect of internal rotation needs to be taken into account. This requires the determination of the methyl internal rotation barrier $V_{3}$, which has been obtained, for each isomer, as the energy difference between the transition state $\left(\angle \mathrm{H} 8-\mathrm{C} 1-\mathrm{C} 2-\mathrm{N} 3=60^{\circ}\right.$, see Figure 2$)$ and the minimum. The equilibrium and $\mathrm{ZPE}$-corrected energies relative to the $E$ isomer are reported in Figure 2. The computed values of $V_{3}$ are given in Table 3 , and are found in excellent agreement with those experimentally evaluated, the discrepancy being well within $2 \%$. The larger value of $V_{3}$ for the $E$ isomer compared to $Z-\mathrm{CH}_{3} \mathrm{CHNH}\left(563.1 \mathrm{~cm}^{-1}\right.$ vs $\left.523.3 \mathrm{~cm}^{-1}\right)$ supports the aforementioned hypothesis that a weak $\mathrm{C}-\mathrm{H} \cdots \mathrm{N}$ hydrogen bond is established in the $E$ isomer. To fully characterize the methyl internal rotation in ethanimine, the moment of inertia of the methyl group around its
$C_{3}$ axis $\left(I_{\alpha}\right)$ has been calculated, resulting in an almost identical value $\left(E: 3.122 \mathrm{u} \AA^{2}\right.$ vs $\left.Z: 3.123 \mathrm{u} \AA^{2}\right)$ for both isomers (see Table 3 ), able to well reproduce the data derived from experiment.

For the fitting procedure, the XIAM package (Hartwig \& Dreizler 1996; Hansen et al. 1999) in conjunction with Watson's A reduced Hamiltonian (Watson 1977) has been used and applied to the newly determined transition frequencies together with the available previous data. The results are collected in Table 3. In addition to the aforementioned $I_{\alpha}$ and $V_{3}$ values, in this Table, the rotational, quartic and sextic centrifugal distortion, and nuclear quadrupole coupling constants are reported together with the computed dipole moment components along the principal axes of inertia. To be directly compared with experiment, the equilibrium values of nuclear quadrupole coupling con- 
stants and dipole moment components have been augmented by vibrational corrections. The latter have been obtained by means of perturbation theory according to Barone (2005). As evident from Table 3, there is an excellent agreement between the computed and experimental rotational constants, with the maximum discrepancy, $0.1 \%$ in relative terms, observed for the $A$ constant. Even if larger discrepancies are noted for the quartic centrifugal distortion constants, dipole moment components, and quadrupole coupling constants, we anyway note a quantitive agreement between theory and experiment. The calculated sextic centrifugal distortion constants have been kept fixed in the fitting procedure because they were found not determinable. Interested readers are referred to Puzzarini et al. (2008) for a statistical analysis of the accuracy of different levels of theory in the determination of rotational constants and to Puzzarini et al. (2012) for their performance in the prediction of centrifugal distortion constants.

Even if not explicitly reported in Table 3, it is interesting to discuss the comparison with the parameters obtained by Lovas et al. (1980), while no comparison can be performed with the most recent study by Loomis et al. (2013) because the details of their fits were not reported. We note that our new measurements did not allow the determination of additional centrifugal distortion constants, but indeed they permit the improvement of all parameters. Particularly noticeable is the revision of the nuclear quadrupole coupling constants of $Z$-ethanimine. By using the newly determined parameters combined with the computed sextic centrifugaldistortion constants, the rotational spectra of both $E$ and $Z$ isomers have been simulated in the $0-500 \mathrm{GHz}$ range at $T=30$ and $100 \mathrm{~K}$, as shown in Figure 3. In the derivation of the rotational transition intensities, the Boltzmann distribution of populations has been considered, with the energy difference between the $E$ and $Z$ isomers taken from Figure 2. These spectral simulations have been carried out using the recently developed rotational spectroscopy module of the Virtual Multifrequency Spectrometer, VMS-ROT (Licari et al. 2017).

\subsection{Infrared Spectroscopy}

Fundamental band frequencies of both isomers have been calculated as described in section 2 . The results are collected in Table 4, where they are compared with the available experimental data from gas-phase (Hashiguchi et al. 1984) and matrix infrared (Stolkin et al. 1977) spectra. This comparison is graphically summarized in Figure 4. In some cases, the original assign- ments were corrected on the basis of our new computed results.

First of all, it is interesting to note that gas-phase and Ar-matrix vibrational frequencies present some important differences. A shift of about $10 \mathrm{~cm}^{-1}$ can be observed for the $\nu_{3}$ and $\nu_{17}$ bands of the $E$ isomer, while for $\nu_{14}$ the disagreement is as large as $19 \mathrm{~cm}^{-1}$. These differences can be explained by the fact that the measurements performed by Stolkin et al. (1977) may be affected by the presence of the noble gas matrix. The latter is expected to produce even more important shifts with respect to gas-phase values for the N-H stretching vibrations, therefore in the following the comparison between theory and experiment will be limited to the spectral region below $3000 \mathrm{~cm}^{-1}$, for which gas-phase data are available as well. In this respect, it should be also recalled that the computed values actually refer to the isolated gas-phase molecule. While a detailed assignment of the vibrational spectrum of ethanimine is beyond the aim of the present work, the theoretical results obtained here show significant disagreements with previous works, and suggest a reassignment of some fundamental bands. In particular, according to Stolkin et al. (1977), the absorptions at $1412 \mathrm{~cm}^{-1}$ and $1392 \mathrm{~cm}^{-1}$ were assigned as the $\nu_{7}$ band of $E$ - and $Z$-ethanimine, respectively. However, on the basis of the CC/DFT predictions, the two assignments need to be exchanged. Even more importantly, the spectral feature at $1252 \mathrm{~cm}^{-1}$ was originally attributed to the $\nu_{8}$ fundamental, while according to our quantum-chemical calculations it has to be attributed to the $\nu_{9}$ normal modes of both isomers, which are expected to overlap, thus giving rise to a very strong band, in agreement with that observed in the Ar-matrix spectrum. According to the re-assignment, the $\nu_{8}$ fundamentals of $E$ - and $Z$-ethanimine produce the strong band measured at $1358 \mathrm{~cm}^{-1}$. Stolkin et al. (1977) identified the bands at $1114 \mathrm{~cm}^{-1}$ and $1106 \mathrm{~cm}^{-1}$ as due to the $\nu_{9}$ bands of $E$ - and $Z$-ethanimine, while calculations indicate that they may arise instead from the $\nu_{15}$ fundamentals of the $Z$ and $E$ isomers, respectively. Nevertheless, it should be noted that the intensity for $\nu_{15}$ of $E$ ethanimine is predicted to be only $0.18 \mathrm{~km} \mathrm{~mol}^{-1}$, while the corresponding band is labeled as medium-strong in the experimental spectrum. Also the assignment of the $\nu_{10}$ and $\nu_{16}$ fundamentals of the $E$ isomer appears interchanged with respect to Stolkin et al. (1977), while that for the $Z$-species has been maintained. However, this is a tentative assignment, in fact three fundamental vibrations (i.e. $\nu_{16}$ of $E$-ethanimine, $\nu_{10}$ and $\nu_{16}$ of $Z$-ethanimine) are predicted to be nearly degenerate (within $2 \mathrm{~cm}^{-1}$ ). These are likely to overlap in the experimental spectrum, as also suggested by the gas-phase 
Table 3. Computed and experimental rotational parameters (Watson A-reduction, values in $\mathrm{MHz}$ ).

\begin{tabular}{|c|c|c|c|c|}
\hline & \multicolumn{2}{|c|}{$E$-ethanimine } & \multicolumn{2}{|c|}{$Z$-ethanimine } \\
\hline & best theoretical ${ }^{a}$ & this work ${ }^{b}$ & best theoretical $^{a}$ & this work ${ }^{b}$ \\
\hline$A_{0}$ & 53178.26 & $53120.561(30)$ & 50002.63 & 49964.87(93) \\
\hline$B_{0}$ & 9780.14 & $9782.7720(47)$ & 9831.98 & $9832.4823(96)$ \\
\hline$C_{0}$ & 8702.82 & $8697.0263(46)$ & 8652.81 & $8646.0305(94)$ \\
\hline$\Delta_{J}$ & $6.48 \times 10^{-3}$ & $6.4641(49) \times 10^{-3}$ & $6.99 \times 10^{-3}$ & $6.938(13) \times 10^{-3}$ \\
\hline$\Delta_{K}$ & 0.568 & $0.5763(34)$ & 0.468 & $0.468^{\mathrm{d}}$ \\
\hline$\Delta_{J K}$ & -0.0165 & $-0.01403(21)$ & -0.0163 & $-0.01219(23)$ \\
\hline$\delta_{J}$ & $1.09 \times 10^{-3}$ & $1.1033(19) \times 10^{-3}$ & $1.25 \times 10^{-3}$ & $1.2657(65) \times 10^{-3}$ \\
\hline$\delta_{K}$ & -0.0535 & $-0.06709(59)$ & -0.0522 & $-0.0642(19)$ \\
\hline$\Phi_{J}$ & $6.18 \times 10^{-9}$ & $6.18 \times 10^{-9 \mathrm{c}}$ & $6.04 \times 10^{-9}$ & $6.04 \times 10^{-9 \mathrm{c}}$ \\
\hline$\Phi_{K}$ & $2.48 \times 10^{-5}$ & $2.48 \times 10^{-5 \mathrm{c}}$ & $2.25 \times 10^{-5}$ & $2.25 \times 10^{-5 \mathrm{c}}$ \\
\hline$\Phi_{J K}$ & $-5.56 \times 10^{-7}$ & $-5.56 \times 10^{-7 \mathrm{c}}$ & $-6.35 \times 10^{-7}$ & $-6.35 \times 10^{-7 \mathrm{c}}$ \\
\hline$\Phi_{K J}$ & $-7.42 \times 10^{-6}$ & $-7.42 \times 10^{-6 \mathrm{c}}$ & $-7.27 \times 10^{-6}$ & $-7.27 \times 10^{-6 \mathrm{c}}$ \\
\hline$\phi_{J}$ & $2.64 \times 10^{-9}$ & $2.64 \times 10^{-9 \mathrm{c}}$ & $2.74 \times 10^{-9}$ & $2.74 \times 10^{-9 \mathrm{c}}$ \\
\hline$\phi_{K}$ & $-2.43 \times 10^{-5}$ & $-2.43 \times 10^{-5 \mathrm{c}}$ & $-2.45 \times 10^{-5}$ & $-2.45 \times 10^{-5 \mathrm{c}}$ \\
\hline$\phi_{J K}$ & $-1.07 \times 10^{-8}$ & $-1.07 \times 10^{-8 \mathrm{c}}$ & $-8.14 \times 10^{-8}$ & $-8.14 \times 10^{-8 \mathrm{c}}$ \\
\hline$\chi_{a a}$ & 1.03 & $0.62(11)$ & -3.62 & $-3.688(13)$ \\
\hline$\chi_{a b}$ & -0.46 & $-0.46^{\mathrm{c}}$ & 1.75 & $1.75^{\mathrm{c}}$ \\
\hline$\chi_{b b}$ & -4.05 & $-3.78(12)$ & 0.57 & $0.548(39)$ \\
\hline$\chi_{c c}$ & 3.02 & $3.16(12)$ & 3.05 & $3.140(39)$ \\
\hline$I_{\alpha}{ }^{\mathrm{d}}$ & 3.122 & $3.1905(17)$ & 3.123 & $3.1700(28)$ \\
\hline$\angle(i, a)^{\mathrm{d}, \mathrm{e}}$ & 23.54 & $24.50(2)$ & 23.61 & $24.19(5)$ \\
\hline$V_{3}{ }^{\mathrm{d}}$ & 563.1 & $566.37(20)$ & 523.3 & $517.41(33)$ \\
\hline$D_{p i 2 J}{ }^{\mathrm{e}}$ & & $0.480(55)$ & & \\
\hline RMS error ${ }^{f}$ & & 0.089 & & 0.039 \\
\hline$\mu_{a}$ & -0.79 & $0.834(23)^{\mathrm{g}}$ & -2.41 & $2.446(4)^{\mathrm{g}}$ \\
\hline$\mu_{b}$ & -1.93 & $1.882(5)^{\mathrm{g}}$ & 0.44 & $0.44^{\mathrm{c}}$ \\
\hline
\end{tabular}

Note-Values in parenthesis denote one standard deviation and apply to the last digits of the constants.

${ }^{a}$ Equilibrium $\mathrm{CCSD}(\mathrm{T}) / \mathrm{CBS}+\mathrm{CV}$ rotational constants augmented by vibrational corrections at the B2PLYP-D3BJ/maug-cc-pVTZ- $d \mathrm{H}$ level. Quartic and sextic centrifugal distortion constants at the CCSD(T)/cc-pCVQZ and B2PLYP-D3BJ/maug-cc-pVTZ- $d \mathrm{H}$ levels, respectively. Dipole moment components (values in debye) and nuclear quadrupole coupling constants at the CCSD(T)/cc-pCVQZ level augmented by vibrational corrections at the B2PLYP-D3BJ/maug-cc-pVTZ- $d \mathrm{H}$ level. The dipole moment along the $c$ axis is null, hence omitted.

${ }^{b}$ Parameters obtained using the XIAM program (Hartwig \& Dreizler 1996; Hansen et al. 1999), the transitions available in literature have been included in the fit.

${ }^{c}$ Fixed at the theoretical value.

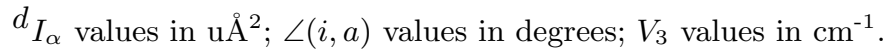

$e^{e}(i, a)$ is the angle between the inertial axis $a$ and the internal rotation axis $i$; $D_{p i 2 J}$ is an internal rotation - overall rotation distortion parameter, see Hansen et al. (1999).

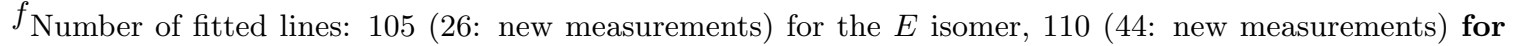
the $Z$ isomer.

$g_{\text {Lovas et al. (1980). Absolute value. }}$ 


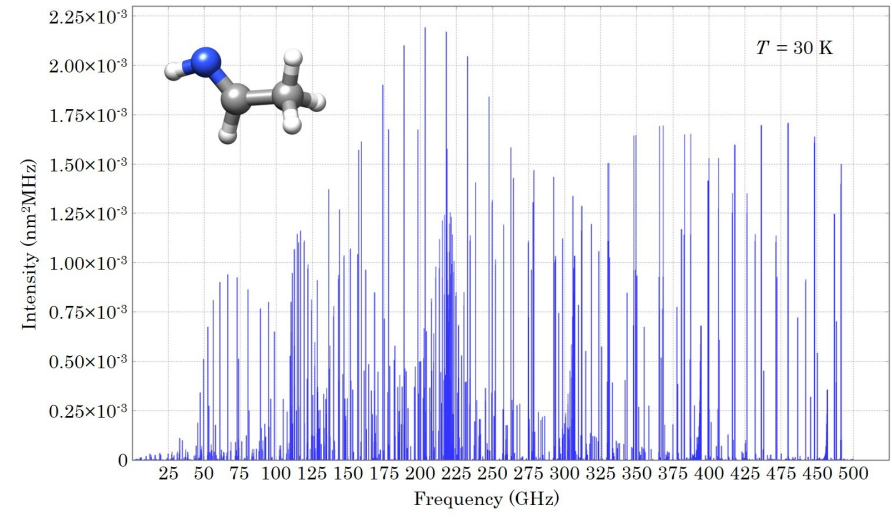

(1a)

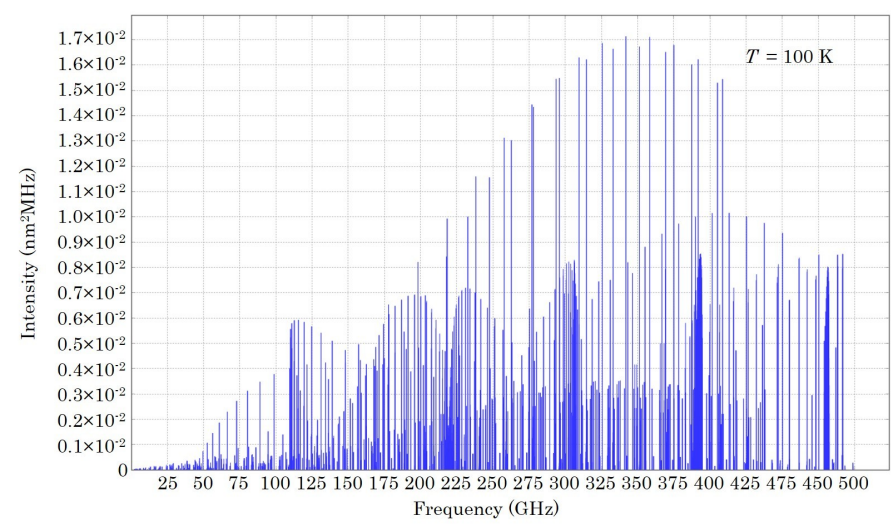

(1b)

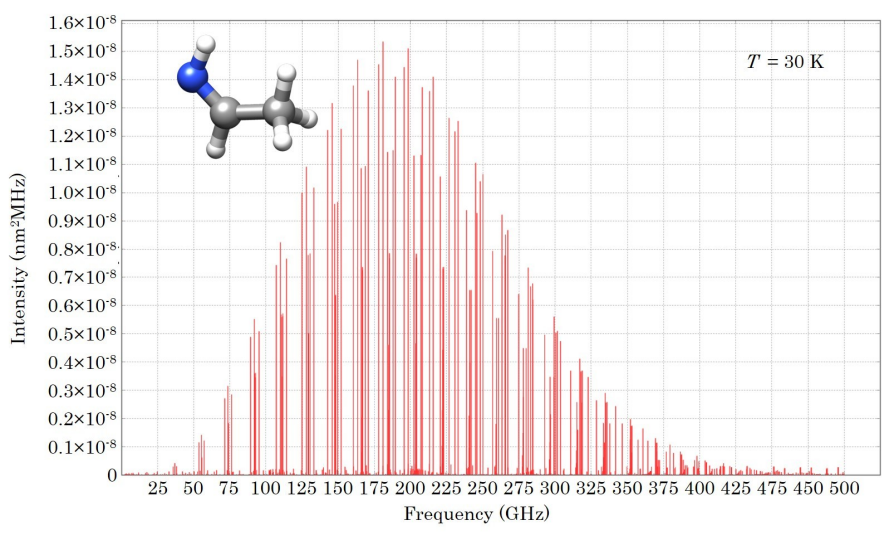

$(2 \mathrm{a})$

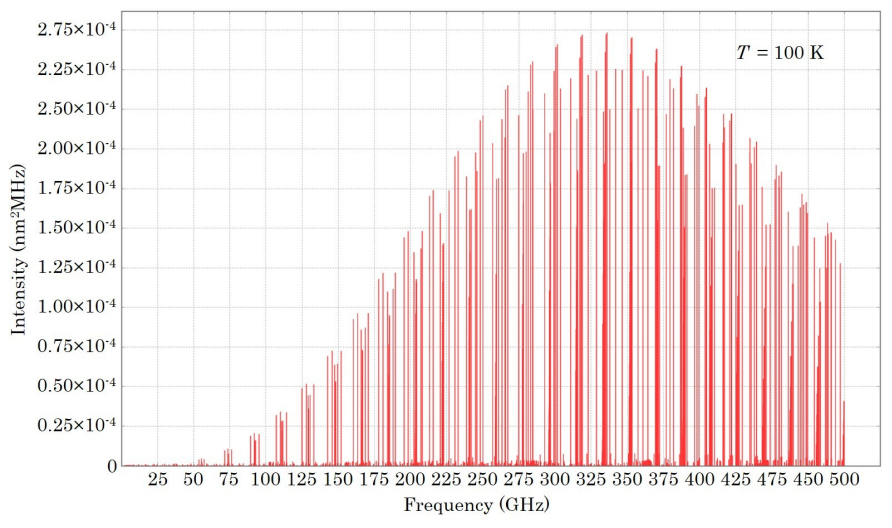

(2b)

Figure 3. Comparison between the simulated rotational spectra for $E$-ethanimine (1a, $1 \mathrm{~b})$ and $Z$-ethanimine (2a, $2 \mathrm{~b})$, obtained using the rotational parameters of Table 3 and without considering nuclear quadrupole coupling. All spectra are scaled according to the Boltzmann distribution. 
Table 4. Comparison between the calculated (harmonic $\omega$, anharmonic $\nu$ ) and the experimental wavenumbers (in $\mathrm{cm}^{-1}$ ) for the fundamental bands. Computed intensities (in $\mathrm{km} / \mathrm{mol}$ ) are also provided.

\begin{tabular}{|c|c|c|c|c|c|c|c|c|c|c|c|}
\hline \multirow{3}{*}{$\begin{array}{l}\text { Symm./ } \\
\text { Mode }\end{array}$} & \multirow{3}{*}{$\begin{array}{l}\text { Approximate } \\
\text { description }\end{array}$} & \multicolumn{5}{|c|}{$E$-ethanimine } & \multicolumn{5}{|c|}{$Z$-ethanimine } \\
\hline & & \multicolumn{3}{|c|}{ Theory } & \multicolumn{2}{|c|}{ Experiment } & \multicolumn{3}{|c|}{ Theory } & \multicolumn{2}{|c|}{ Experiment } \\
\hline & & $\omega^{\mathrm{a}}$ & $\nu^{\mathrm{b}}$ & $I_{\mathrm{anh}}{ }^{\mathrm{c}}$ & Exp. $1^{\mathrm{d}}$ & Exp. $2^{\mathrm{e}}$ & $\omega^{\mathrm{a}}$ & $\nu^{\mathrm{b}}$ & $I_{\mathrm{anh}}{ }^{\mathrm{c}}$ & Exp. $1^{\mathrm{d}}$ & Exp. $2^{\mathrm{e}}$ \\
\hline \multicolumn{12}{|l|}{$\mathrm{A}^{\prime}$} \\
\hline$\nu_{1}$ & $\mathrm{~N}-\mathrm{H}$ stretch & 3468 & 3300 & 1.96 & $3247(\mathrm{vvw})$ & - & 3425 & 3254 & 5.63 & $3264(\mathrm{vvw})$ & - \\
\hline$\nu_{2}$ & $\mathrm{CH}_{3}$ asym. stretch & 3156 & 3015 & 8.62 & $3018(\mathrm{~m})$ & - & 3134 & 2991 & 24.79 & $2990(\mathrm{~m})$ & 2988 \\
\hline \multirow[t]{2}{*}{$\nu_{3}$} & $\mathrm{CH}_{3}$ sym. stretch $(\mathrm{E})$ & 3048 & 2941 & 7.11 & $2925(\mathrm{~ms})$ & 2916 & 3097 & 2948 & 23.71 & $2954(\mathrm{~ms})$ & \\
\hline & $\mathrm{CH}$ stretch $(\mathrm{Z})$ & & & & & & & & & & \\
\hline \multirow[t]{2}{*}{$\nu_{4}$} & $\mathrm{CH}$ stretch $(\mathrm{E})$ & 3039 & 2881 & 47.59 & $2885(\mathrm{~m})$ & 2886 & 3039 & 2936 & 4.42 & - & - \\
\hline & $\mathrm{CH}_{3}$ sym. stretch $(\mathrm{Z})$ & & & & & & & & & & \\
\hline$\nu_{5}$ & $\mathrm{C}=\mathrm{N}$ stretch $+\mathrm{C}-\mathrm{C}$ stretch & 1709 & 1665 & 66.81 & $1652(\mathrm{vs})$ & 1655 & 1706 & 1663 & 62.41 & $1659(\mathrm{sh})$ & 1651 \\
\hline$\nu_{6}$ & $\mathrm{CH}_{3}$ asym. bend & 1483 & 1442 & 16.70 & $1438(\mathrm{~ms})$ & - & 1490 & 1449 & 21.18 & $1438(\mathrm{~ms})$ & - \\
\hline$\nu_{7}$ & $\mathrm{CNH}$ bend & 1436 & 1395 & 12.57 & $1392(\mathrm{~m})$ & - & 1435 & 1408 & 18.43 & $1412(\mathrm{~m})$ & - \\
\hline$\nu_{8}$ & $\mathrm{CH}_{3}$ sym. bend & 1395 & 1362 & 13.02 & $1358(\mathrm{~s})$ & - & 1405 & 1371 & 8.34 & $1358(\mathrm{~s})$ & - \\
\hline$\nu_{9}$ & HNCH scissor & 1280 & 1250 & 28.53 & $1252(\mathrm{vs})$ & 1250 & 1285 & 1251 & 60.80 & $1252(\mathrm{vs})$ & 1250 \\
\hline$\nu_{10}$ & $\mathrm{CNH}$ ip bend ${ }^{f}+\mathrm{C}-\mathrm{C}$ stretch & 1067 & 1044 & 32.34 & $1045(\mathrm{vs})$ & 1045 & 1075 & 1051 & 18.51 & $1040(\mathrm{~s})$ & - \\
\hline$\nu_{11}$ & $\mathrm{C}-\mathrm{C}$ stretch & 933 & 924 & 6.88 & $950(\mathrm{w})$ & - & 918 & 910 & 3.40 & $920(w m)$ & - \\
\hline$\nu_{12}$ & NCC bend & 488 & 488 & 17.24 & $485(\mathrm{~s})$ & - & 490 & 491 & 7.55 & 498(wm) & - \\
\hline \multicolumn{12}{|l|}{$\mathrm{A}^{\prime \prime}$} \\
\hline$\nu_{13}$ & $\mathrm{CH}_{3}$ asym. stretch & 3109 & 2967 & 13.97 & - & - & 3111 & 2969 & 11.02 & - & - \\
\hline$\nu_{14}$ & $\mathrm{CH}_{3}$ asym. bend & 1489 & 1444 & 8.79 & $1435(\mathrm{~s})$ & 1454 & 1483 & 1438 & 8.82 & $1435(\mathrm{~s})$ & 1454 \\
\hline \multirow[t]{2}{*}{$\nu_{15}$} & $\mathrm{HNCH}$ twist (E) & 1122 & 1095 & 0.18 & $1106(\mathrm{~ms})$ & - & 1142 & 1114 & 37.22 & $1114(\mathrm{~ms})$ & - \\
\hline & $\mathrm{HNCH}$; oop bend $(\mathrm{Z})^{f}$ & & & & & & & & & & \\
\hline$\nu_{16}$ & $\mathrm{CH}_{3}$ asym. bend $+\mathrm{N}-\mathrm{H}$ oop bend & 1081 & 1050 & 15.04 & $1052(w m)$ & - & 1077 & 1049 & 14.39 & $1040(\mathrm{~s})$ & - \\
\hline \multirow[t]{2}{*}{$\nu_{17}$} & $\mathrm{HNCH}$ oop bend $(\mathrm{E})^{f}$ & 680 & 679 & 39.69 & $668(\mathrm{~s})$ & 678 & 691 & 686 & 6.77 & $674(\mathrm{w})$ & 654 \\
\hline & HNCH twist (Z) & & & & & & & & & & \\
\hline$\nu_{18}$ & $\mathrm{CH}_{3}$ torsion & 187 & 171 & 2.19 & - & - & 175 & 158 & 0.25 & - & - \\
\hline
\end{tabular}

Note-Values in $\mathrm{cm}^{-1}$; w: weak, m: medium, s: strong, v: very.

${ }^{a}$ Harmonic: $\operatorname{CCSD}(\mathrm{T}) / \mathrm{cc}-\mathrm{pCVQZ}$.

${ }^{b}$ Harmonic: CCSD(T)/cc-pCVQZ; anharmonic: B2PLYP-D3BJ/maug-cc-pVTZ- $d$ H. See text.

${ }^{c}$ Anharmonic IR intensities at the B2PLYP-D3BJ/maug-cc-pVTZ- $d$ H level.

$d_{\text {Stolkin et al. (1977). }}$

${ }^{e}$ Hashiguchi et al. (1984).

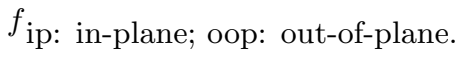


measurements which report only one band at $1045 \mathrm{~cm}^{-1}$, that may be split in the Ar-matrix spectra because of matrix effects. On the basis of the computed intensities the bands measured at $668 \mathrm{~cm}^{-1}$ and $674 \mathrm{~cm}^{-1}$ have to be ascribed to the $\nu_{17}$ vibrations of $E$ - and $Z$ ethanimine, respectively, again reversed with respect to the assignment in the original work. The above discussion clearly suggests that a careful reanalysis of the experimental infrared spectra of ethanimine is strongly required, which may be complemented by new measurements to overcome some of the uncertainty raising from those currently available. For this reason, a more detailed theoretical investigation, also dealing with combination and overtone bands as well as isotopically substituted species, will be the subject of a dedicated work.

\section{ASTROPHYSICAL IMPLICATIONS}

According to the combined crossed-beam and theoretical study on the formation of nitriles and imines in the atmosphere of Titan reported in Balucani et al. (2010), ethanimine is expected to be the second most important molecular product of the reaction of excited nitrogen atoms $\mathrm{N}\left({ }^{2} \mathrm{D}\right)$ with ethane. $\mathrm{CH}_{3} \mathrm{CHNH}$ can further react and be thus a source of nitrogen-rich molecules and aerosols via addition reactions. It can also undergo UV photodissociation, thus leading to the formation of reactive radicals that can enhance the formation of nitrogenrich complex species. To confirm the reliability of the suggested formation route of ethanimine in Titan's atmosphere, its detection is decisive. It is in the context of supporting its millimeter/submillimeterwave and/or infrared spectral observations that lies the importance of the present spectroscopic characterization.

As far as the millimeter/submillimeter-wave frequency domain is concerned, an opportunity is offered by the ground-based observatory ALMA that combines high sensitivity with high spectral and spatial resolution interferometric observations. The ALMA's working frequency range (31-950 GHz) points out the importance of the accurate predictions for rotational transitions lying at frequencies higher than $150 \mathrm{GHz}$ provided by the present study. Cordiner et al. (2015) and Palmer et al. (2017) demonstrated the viability of using data from ALMA to detect prebiotic species in Titan's atmosphere by reporting the first spectroscopic detection of rotational lines of ethyl cyanide and vinyl cyanide, respectively, in the $222-240 \mathrm{GHz}$ frequency range. In this frequency range, both isomers of $\mathrm{CH}_{3} \mathrm{CHNH}$ show some intense lines that deserve further investigation. By way of exam- ple, we report below a couple of them for both isomeric species:

- $E$ isomer $12_{1,12}-\mathbf{1 1}_{0,11}$ : 232686.59(7) $\mathrm{MHz} \quad E_{u}=40.24 \mathrm{~cm}^{-1}$ $\mathbf{1 4}_{0,14}-\mathbf{1 3}_{1,13}$ : 238199.37(3) MHz $E_{u}=55.73 \mathrm{~cm}^{-1}$

- $Z$ isomer $\mathbf{1 3}_{1,13}-\mathbf{1 2}_{1,12}$ : $230701.52(6) \mathrm{MHz} \quad E_{u}=47.72 \mathrm{~cm}^{-1}$ $1 \mathbf{3}_{0,13}-\mathbf{1 2}_{0,12}$ : 232913.90(5) $\mathrm{MHz} \quad E_{u}=47.31 \mathrm{~cm}^{-1}$

where $E_{u}$ is the upper state (in emission) energy level.

Concerning infrared spectral observations, we note that the analysis of the IR spectra recorded by Cassini CIRS instrument $\left(10-1500 \mathrm{~cm}^{-1}\right)$ is not a valuable route because the strongest vibrational features of ethanimine potentially present in the spectra are covered by strong absorptions of ethylene $\left(\mathrm{C}_{2} \mathrm{H}_{2}\right)$ and methane $\left(\mathrm{CH}_{4}\right)$. We only point out that the modelling of the CIRS spectrum with a radiative transfer model not accounting for $\mathrm{CH}_{3} \mathrm{CHNH}$ shows an unassigned transition at $\sim 680$ $\mathrm{cm}^{-1}$ in the residuals, i.e., in the observed-calculated differences (Coustenis et al. 2007), which might correspond to the $\nu_{17}$ band of the $E$ isomer. However, the best opportunity to clarify this point and to attempt a detection in Titan's atmosphere by using IR spectroscopy will be provided by the James Webb Space Telescope (JWST) that will cover the 0.6-29 $\mu \mathrm{m}$ wavelength range $\left(16667-345 \mathrm{~cm}^{-1}\right)$. Mention of the high-resolution EXES instrument mounted on Stratospheric Observatory for Infrared Astronomy (SOFIA) in the mid-infrared region (4.5-28.3 $\mu \mathrm{m}$ wavelength range, $2222-353 \mathrm{~cm}^{-1}$ wavenumber range) is also here deserved. In this respect, thanks to our revision of the infrared spectrum of both isomeric species, accurate predictions are now available to guide the search of ethanimine in Titan's and other planetary atmospheres. According to our investigation, the most suitable band is the $\nu_{5}$ fundamental lying at 1665 $\mathrm{cm}^{-1}$ for $E-\mathrm{CH}_{3} \mathrm{CHNH}$ and at $1663 \mathrm{~cm}^{-1}$ for the $Z$ isomer. The mid-IR instrument (MIRI) on board the JWST has a resolving power $R$ ranging from 2400 to 3700 in the 5 to $7.7 \mu \mathrm{m}$ range, thus not disentangling the two features. However, at high resolution the $\nu_{5}$ band is expected to provide a characteristic feature showing an asymmetric and partially resolved doublet, where the asymmetry in intensity is due to the different population ratio, which in turn is related to the 


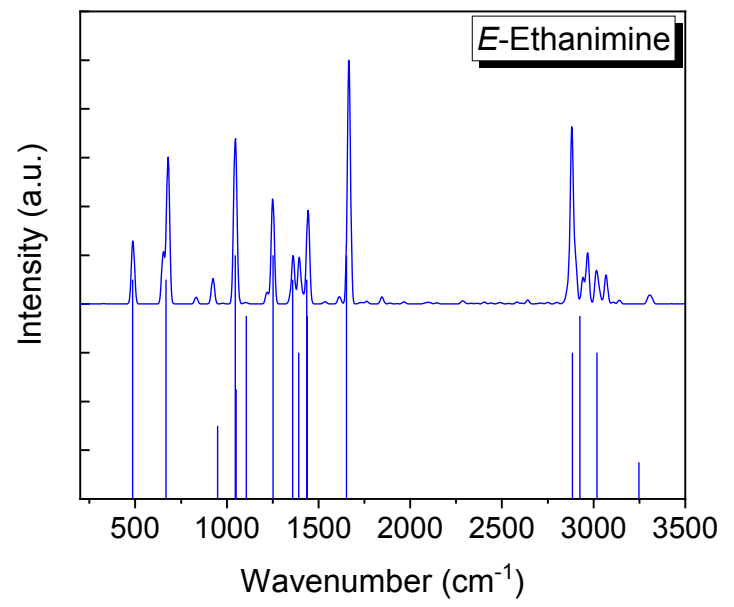

(a)

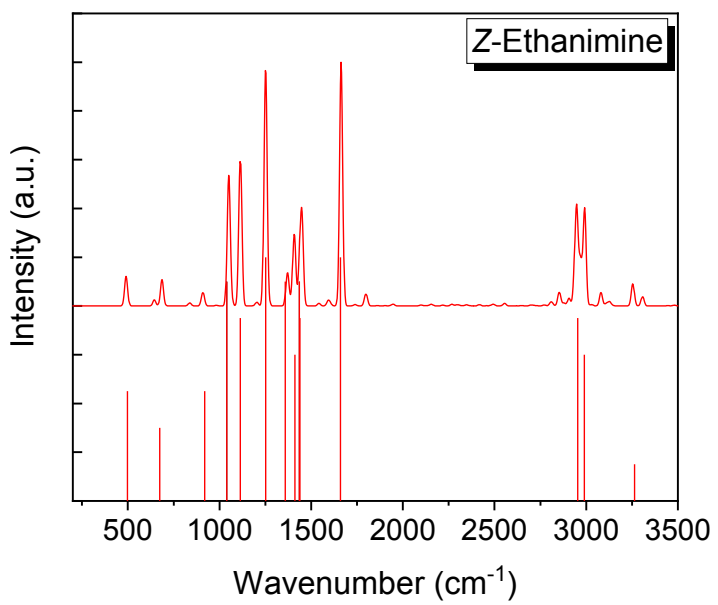

(b)

Figure 4. Simulated infrared spectra for E-ethanimine (a) and $Z$-ethanimine (b) obtained with anharmonic wavenumbers from hybrid CCSD(T)/DFT computations and intensities at the B2PLYP-D3/maug-cc-pVTZ$d \mathbf{H}$ level. Computed values have been convoluted with a Gaussian function (half-width at half-maximum $=10$ $\mathbf{c m}^{-1}$ ). Stick spectra represent experimental values from Ar-matrix measurements (Stolkin et al. 1977); for the intensity the following equivalence with respect to the qualitative description was applied: very strong $=1$; strong $=0.9$; medium-strong $=0.75 ;$ medium $=0.6$; weak-medium $=0.45$; weak $=0.3$; very very weak $=0.15$.

atmosphere's temperature. Other intense features are the $\nu_{3}$ band of $E$-ethanimine at 2881 $\mathrm{cm}^{-1}$ and the $\nu_{9}$ fundamental of the $Z$ isomer, which is almost overlapped with that of the $E$ species.

Indeed, astronomical line searches are not only limited to Titan's atmosphere, but the potential astrophysical objects span from protostellar envelopes and protoplanetary disks to the atmospheres of exoplanets. As mentioned in the Introduction, both isomers of ethanimine have been identified in a survey of $\operatorname{SgrB} 2(\mathrm{~N})$ in the frame of the PRIMOS project (Loomis et al. 2013). This detection in the centimeter-wave spectral window calls for further searches at higher frequencies, in the millimeter/submillimeter-wave spectral range, in regions forming future Sun-like stars. Therefore, to detect ethanimine in these regions, line emissions in the millimeter/submillimeter-wave spectral range are instructive tracers. As mentioned above, Figure 3 provides an overview of the rotational spectra up to $500 \mathrm{GHz}$ frequency range for the $E$ and $Z$ isomers, respectively, at two different temperatures: $T=30 \mathrm{~K}$ and $100 \mathrm{~K}$. These have been chosen as representative temperatures for starless and dense core regions and for the regions around protostars and outflow shocks, respectively. It is observed that, for both $E$ - and $Z-\mathrm{CH}_{3} \mathrm{CHNH}$, at $T=30 \mathrm{~K}$ the spectra show the maximum intensity at $\sim 200 \mathrm{GHz}$. Moving to $T=100 \mathrm{~K}$, the maximum shifts at higher frequency, $\sim 330-350 \mathrm{GHz}$, with the intensity increasing by one order of magnitude for the $E$ isomer and by four orders of magnitude for $Z$-ethanimine. Opportunities can be provided by spectral surveys like those that can be collected using single-dish telescopes (like IRAM 30-m radiotelescope in Pico Veleta) as well as interferometers (like NOEMA, NOrthern Extended Millimeter Array on the French Alps, and ALMA). To give an example in this context, the large program $\mathrm{ASAI}^{1}$ data have been recently used for searching $\mathrm{C}$-cyanomethanimine emission toward nearby Sun-like-star forming regions (Melosso et al. 2017). In this respect, the ASAI data from the $1.3 \mathrm{~mm}$ receiver of the Pico Veleta 30-m single-dish (200-276 GHz) can be considered a good opportunity for searching the two isomeric $\mathrm{CH}_{3} \mathrm{CHNH}$ species, because both of them present several bright transitions in that frequency range.

\section{CONCLUDING REMARKS}

In the present work, aiming at providing useful information to guide astronomical searches for ethanimine, an accurate computational spectroscopy characterization, combined with new measurements for the rotational spectra of both isomers, has been accomplished.

\footnotetext{
${ }^{1}$ http://www.oan.es/asai/
} 
As pointed out along the manuscript, the computational approaches employed allowed us to complement experiment in the field of rotational spectroscopy and to revise the infrared spectra assignments, thus providing accurate data to guide detection in space. Ultimately, we are confident that the present study can provide useful information for shedding light on prebiotic chemistry in space or planetary atmospheres.

This work has been supported by MIUR 'PRIN 2015' funds (project "STARS in the CAOS (Simulation Tools for Astrochemical Reactivity and Spectroscopy in the Cyberinfrastructure for Astrochemical Organic
Species)" - Grant Number 2015F59J3R), by the University of Bologna (RFO funds), and by Scuola Normale Superiore (funds for project GR16_B_TASINATO, COSMO: "Combined experimental and computational spectroscopic modeling for astrochemical applications"). The support of the COST CMTS-Actions CM1405 (MOLIM: MOLecules In Motion) and CM1401 (Our Astro-Chemical History) is also acknowledged. CP deeply thanks Dr. Claudio Codella for useful discussions.

Software: Gaussian16 (Frisch et al. 2016), CFOUR (Stanton et al. 2008), VMS (Licari et al. 2017), XIAM (Hartwig \& Dreizler 1996; Hansen et al. 1999).

\section{REFERENCES}

Balucani, N., Leonori, F., Petrucci, R., et al. 2010, Faraday Discuss., 147, 189

Barone, V. 2005, J. Chem. Phys., 122, 014108

Barone, V., Biczysko, M., Bloino, J., et al. 2015a, J. Chem.

Theo. Comput., 11, 4342

Barone, V., Biczysko, M., Bloino, J., \& Puzzarini, C. 2013, Phys. Chem. Chem. Phys., 15, 11094

—. 2014, J. Chem. Phys., 141, 034107

Barone, V., Biczysko, M., \& Puzzarini, C. 2015b, Acc. Chem. Res., 48, 1413

Bloino, J., Baiardi, A., \& Biczysko, M. 2016, Int. J. Quantum Chem., 116, 1543

Bloino, J., \& Barone, V. 2012, J. Chem. Phys., 136, 124108

Bloino, J., Biczysko, M., \& Barone, V. 2012, J. Chem.

Theo. Computat., 8, 1015

Brown, R., Lebreton, J. P., \& Waite, J. 2010, Titan from Cassini-Huygens (Springer Netherlands)

Brown, R. D., Godfry, P. D., \& Winkler, D. A. 1980, Austr. J. Chem., 33, 1

Cordiner, M. A., Palmer, M. Y., Nixon, C. A., et al. 2015, ApJL, 800, arXiv:1410.5325

Coustenis, A., Achterberg, R. K., Conrath, B. J., et al. 2007, Icarus, 189, 35

Degli Esposti, C., Dore, L., \& Bizzocchi, L. 2014, A\&A, 565, A66

Degli Esposti, C., Dore, L., Melosso, M., et al. 2017, ApJS, 230, $7 \mathrm{pp}$

Dore, L., Bizzocchi, L., \& Degli Esposti, C. 2012, A\&A, 544, A19

Dunning, T. H. J. 1989, J. Chem. Phys., 90, 1007

Durig, J. R., Zhou, S. X., Zhou, C. X., \& Durig, N. E. 2010, J. Mol. Struct., 967, 1

Elsila, J. E., Dworkin, J. P., Bernstein, M. P., Martin, M. P., \& Sandford, S. A. 2007, ApJ, 660, 911
Feller, D. 1993, J. Chem. Phys., 98, 7059

Frisch, M. J., Trucks, G. W., Schlegel, H. B., et al. 2016, Gaussian 16 Revision A.03, , , gaussian Inc. Wallingford CT

Gauss, J., \& Stanton, J. 1997, Chem. Phys. Lett., 276, 70

Goerigk, L., \& Grimme, S. 2011, J. Chem. Theo. Computat., 7, 291

Gordy, W., \& Cook, R. L. 1984, Microwave Molecular Spectra, 3rd edn. (John Wiley \& Sons)

Grimme, S. 2006, J. Chem. Phys., 124, 034108

Grimme, S., Ehrlich, S., \& Goerigk, L. 2011, J. Computat. Chem., 32, 1456

Hansen, N., Mäder, H., \& Bruhn, T. 1999, Mol. Phys., 97, 587

Hartwig, H., \& Dreizler, H. 1996, Z. Naturforsch., 51a, 923

Hashiguchi, K., Hamada, Y., Tsuboi, M., Koga, Y., \& Kondo, S. 1984, J. Mol. Spectrosc., 105, 81

Heckert, M., Kàllay, M., \& Gauss, J. 2005, Mol. Phys., 103, 2109

Heckert, M., Kàllay, M., Tew, D. P., Klopper, W., \& Gauss, J. 2006, J. Chem. Phys., 125

Helgaker, T., Klopper, W., Koch, H., \& Noga, J. 1997, J. Chem. Phys., 106, 9639

Herbst, E., \& van Dishoeck, E. F. 2009, Ann. Rev. Astron. Astrophys., 47, 427

Licari, D., Tasinato, N., Spada, L., Puzzarini, C., \& Barone, V. 2017, Journal of Chemical Theory and Computation, 13, 4382

Loomis, R. A., Zaleski, D. P., Steber, A. L., et al. 2013, ApJ Lett., 765, 7

Lovas, F. J., Suenram, R. D., Johnson, D. R., Clark, F. O., \& Tiemann, E. 1980, J. Chem. Phys., 72, 4964

Melosso, M., Melli, A., Puzzarini, C., et al. 2017, A\&A, doi:10.1051/0004-6361/201731972 
Mendolicchio, M., Penocchio, E., Licari, D., Tasinato, N., \& Barone, V. 2017, J. Chem. Theo. Comput., 13, 3060

Mills, I. M. 1972, in Molecular Spectroscopy: Modern Research, ed. K. N. Rao \& C. W. Mathews (Academic Press, New York), 115-140

Müller, H. S. P., Schlöder, F., Stutzki, J., \& Winnewisser, G. 2005, J. Mol. Struct., 742, 215

Müller, H. S. P., Thorwirth, S., Roth, D. A., \& Winnewisser, G. 2001, A\&A, 370, L49

Palmer, M. Y., Cordiner, M. A., Nixon, C. A., et al. 2017, Science Advances, 3, doi:10.1126/sciadv.1700022

Papajak, E., Leverentz, H. R., Zheng, J., \& Truhlar, D. G. 2009, J. Chem. Theo. Computat., 5, 1197

Piccardo, M., Bloino, J., \& Barone, V. 2015, Int. J. Quantum Chem., 115, 948

Puzzarini, C. 2013, Phys. Chem. Chem. Phys., 6595

—. 2015, J. Phys. Chem. A, 119, 11614

—. 2017, Int. J. Quantum Chem., 117, 129

Puzzarini, C., Ali, A., Biczysko, M., \& Barone, V. 2014, ApJ, 792, 118

Puzzarini, C., Biczysko, M., \& Barone, V. 2010a, J. Chem. Theory Comput., 6, 828

—. 2011, J. Chem. Theory Comput., 7, 3702

Puzzarini, C., Cazzoli, G., \& Gauss, J. 2012, J. Chem. Phys., 137, 154311

Puzzarini, C., Heckert, M., \& Gauss, J. 2008, J. Chem. Phys., 128

Puzzarini, C., Stanton, J. F., \& Gauss, J. 2010b, Int.l Rev. Phys. Chem., 273

Quan, D., Herbst, E., Corby, J. F., Durr, A., \& Hassel, G. 2016, ApJ, 824, 14
Raghavachari, K., Trucks, G. W., Pople, J. A., \& Head-Gordon, M. 1989, Chem. Phys. Lett., 157, 479

Schneider, W., \& Thiel, W. 1989, Chem. Phys. Lett., 157, 367

Schuurman, M. S., Allen, W. D., von Ragué Schleyer, P., \& Schaefer III, H. F. 2005, J. Chem. Phys., 122, 104302

Shemansky, D. E., Stewart, A. I. F., West, R. A., et al. 2005, Science, 308, 978

Spada, L., Tasinato, N., Bosi, G., et al. 2017, Journal of Molecular Spectroscopy, 337, 90 , spectroscopy and Inter/Intramolecular Dynamics in Honor of Walther Caminati - Part 2

Stanton, J. F., Gauss, J., Harding, M. E., \& Szalay, P. G. 2008, CFOUR, Coupled-Cluster techiques for Computational Chemistry, v1.0, for the current version, see http://www.cfour.de.

Stolkin, I., Ha, T.-K., \& Gnthard, H. 1977, Chem. Phys., 21,327

Tennyson, J. 2005, Astronomical Spectroscopy (London: Imperial College Press)

Tielens, A. G. G. M. 2013, Rev. Mod. Phys., 85, 1021

Tinetti, G., Encrenaz, T., \& Coustenis, A. 2013, Astron. Astrophys. Rev., 21, 63

Vazart, F., Calderini, D., Puzzarini, C., Skouteris, D., \& Barone, V. 2016, J. Chem. Theo. Comput., 12, 5385

Watson, J. K. 1977, Vibrational spectra and structure, 6, 1 Woon, D. E. 2002, ApJ Lett., 571, L177

Woon, D. E., \& Dunning, T. H. J. 1995, J. Chem. Phys., 103,4572 